\title{
Comportamento de Lutzomyia longipalpis, vetor principal da leishmaniose visceral americana, em Campo Grande, Estado do Mato Grosso do Sul
}

\author{
Behavior of Lutzomyia longipalpis, the main vector of American visceral \\ leishmaniasis, in Campo Grande, State of Mato Grosso do Sul
}

\author{
Elaine Araujo e Silva ${ }^{1}$, Renato Andreotti² e Michael Robin Honer ${ }^{3}$
}

\begin{abstract}
RESUMO
O município de Campo Grande, Estado de Mato Grosso do Sul, Brasil, apresenta ocorrência de casos humanos e caninos de leishmaniose visceral desde 2002 e é classificado como área com transmissão intensa. O estudo foi realizado no período de maio de 2003 a abril de 2005 , em parceria com a Fundação Nacional de Saúde e a Secretaria de Saúde do Estado, com o objetivo de conhecer o comportamento e a sazonalidade da espécie Lutzomyia longipalpis. As capturas foram realizadas com armadilhas luminosas, tipo CDC, em doze estações distribuídas na zona urbana. As estações com maior densidade situam-se na parte sul da cidade e a abundância relativa aumentou durante, ou logo após, as precipitações pluviométricas. Nos meses frios e secos a quantidade foi reduzida e a abundância relativa foi maior no peridomicílio. A borrifação com alphacypermetrina, em intervalos de quatro meses, contribuiu para a diminuição do vetor em três das quatro estações borrifadas e, das oito que não sofreram intervenção química, cinco tiveram aumento.
\end{abstract}

Palavras-chaves: Leishmania chagasi. Leishmaniose visceral. Urbanização. Campo Grande. Mato Grosso do Sul.

\section{ABSTRACT}

The municipality of Campo Grande, State of Mato Grosso do Sul, Brazil, has presented cases of canine and human visceral leishmaniasis since 2002, and is classified as an area of intense transmission. This study was carried out from May 2003 to April 2005, in partnership with the National Health Foundation and the State Health Department, with the objective of determining the behavior and seasonality of the species Lutzomyia longipalpis. Captures were accomplished using luminous traps of CDC type, at twelve stations distributed in the urban zone. The stations with the highest population densities were situated in the southern part of the city and the relative abundance increased during or immediately after rainfall. During the cold and dry months, the number of specimens was reduced and the relative abundance was higher in babitats surrounding homes. Spraying with alpha-cypermethrin at four-month intervals contributed towards reducing the vector numbers in three of the four sprayed stations. Among the eight stations that did not receive chemical interventions, five showed an increase.

Key-words: Leishmania chagasi. Visceral leishmaniasis. Urbanization. Campo Grande. Mato Grosso do Sul.

A leishmaniose visceral (LV) é uma zoonose de grande importância para a saúde pública e para a medicina veterinária, com distribuição mundial, atingindo 88 países. Mais de $90 \%$ dos casos ocorrem em Bangladesh, Brasil, Índia e Sudão $0^{28}$.

A transmissão ocorre através da picada do flebotomíneo da espécie Lutzomyia longipalpis, díptero da família Psychodidae, principal vetor da leishmaniose visceral no Novo Mundo ${ }^{29}$. Santos e $\operatorname{cols}^{26}$, em 1998, consideraram a espécie Lutzomyia cruzi nos municípios de Ladário e Corumbá, MS como vetor da LV; para outros autores ${ }^{15}$, existem dúvidas devido à existência de Lutzomyia longipalpis na área, fato relatado por Santos e cols², em 2003.

Na América Latina, a LV possui uma ampla distribuição, estendendo-se desde o México até a Argentina. Após 1984, estimativas mostravam que $90 \%$ dos casos registrados no Novo Mundo eram do Brasil ${ }^{15}$. Em 2002, houve o relato de sete casos de leishmaniose cutânea e um caso de leishmaniose visceral na Cidade de Sincelejo, Colômbia 5 .

\footnotetext{
1. Centro de Controle de Zoonoses, Prefeitura Municipal de Campo Grande, MS. 2. EMBRAPA - Empresa Brasileira de Pesquisa Agropecuária, Campo Grande, MS. 3. Universidade Católica Dom Bosco, Campo Grande, MS.

Endereço para correspondência: Dr. Renato Andreotti. EMBRAPA Gado de Corte. Br 262 Km 04, Caixa Postal 154, $79002-970$ Campo Grande, MS

Tel: 5567 3368-2178; Fax: 5567 3368-2150

e-mail: andreott@cnpgc.embrapa.br

Recebido em: 05/12/2006

Aceito em: 20/06/2007
} 
A Leishmaniose visceral é uma doença reemergente, que apresenta expansão geográfica e processo de urbanização em várias regiões do Brasil ${ }^{16}$. Os Estados de Alagoas, Bahia, Ceará, Distrito Federal, Espírito Santo, Goiás, Maranhão, Mato Grosso ${ }^{19}$, Mato Grosso do Sul, Minas Gerais, Pará, Paraíba, Pernambuco, Piauí, Rio de Janeiro, Rio Grande do Norte, Roraima, Sergipe, São Paulo e Tocantins possuem casos notificados ${ }^{15}$. Em 2003, os Estados que apresentaram maiores taxas de incidência foram Tocantins, Piauí, Maranhão, Mato Grosso do Sul e Roraima ${ }^{18}$. Municípios como Araçatuba, $\mathrm{SP}^{10}$, Teresina, $\mathrm{PI}^{2}$, Belo Horizonte ${ }^{1624}$ e Porteirinha, $\mathrm{MG}^{4}$, Três Lagoas e Campo Grande, MS, Palmas, $\mathrm{TO}^{18}$, tem enfrentado epidemias.

0 padrão de transmissão da doença pode se modificar devido às alterações ambientais e, consequientemente, à adaptação do vetor, associado às migrações da populaçã $0^{517}$.

O Estado de Mato Grosso do Sul sofreu, nos últimos anos, modificações ambientais que podem ter contribuído para a disseminação do vetor, como a construção de um gasoduto e a destruição de áreas do cerrado. No Município de Campo Grande, a abertura de avenidas acompanhando os cursos das águas e a derrubada da vegetação para construção de casas populares foram fatores de mudança do ambiente.

Os primeiros casos caninos surgiram em 1998, sendo isolada Leishmania chagasi ${ }^{27}$, e os primeiros casos humanos foram notificados em 2002 (com início dos sintomas em 2001). Também no ano de 2002, foram notificados mais 19 casos autóctones, sendo o município classificado como área de transmissão intensa. Em 2005, foram confirmados 153 casos humanos e 6.870 casos caninos $^{23}$. 0 monitoramento apresentado aqui, foi iniciado em maio de 2003, em conjunto com a Fundação Nacional de Saúde e a Secretaria de Estado de Saúde, com o objetivo de conhecer a sazonalidade e o comportamento da espécie Lutzomyia longipalpis, no município de Campo Grande.

\section{MATERIAL E MÉTODOS}

Área de estudo. 0 município de Campo Grande é extenso, com uma área de $8.096 \mathrm{~km}^{2}$ e uma população estimada para 2005 de 749.768 habitantes, segundo o Instituto Brasileiro de Geografia e Estatística (IBGE) ${ }^{14}$. Possui altitude aproximada de $530 \mathrm{~m}$ acima do nível do mar e suas coordenadas geográficas são $20^{\circ} 27$ '5,5" latitude sul e 54³7'25,7" longitude oeste. Segundo a classificação de Köppen, o clima é do tipo Aw, tropical úmido com estação chuvosa no verão e seca no inverno. 0 período de maior precipitação pluviométrica vai de outubro a abril, quando a temperatura média oscila em torno de $24^{\circ} \mathrm{C}$. 0 s meses de menor precipitação são: junho, julho e agosto e a temperatura média é de $20^{\circ} \mathrm{C}$.

0 município encontra-se localizado na Bacia Hidrográfica do Rio Paraná, com exceção de uma pequena porção a noroeste situada na Bacia Hidrográfica do Rio Paraguai, na qual encontram-se os córregos Mateira, Ceroula e Angico. 0 Rio Anhanduí é o principal curso d'água do município, possuindo numerosos afluentes. Os córregos Guariroba, Água Turva, Estaca e Ribeirão das Botas se originam da subbacia do Rio Pardo que é afluente do Rio Paraná.
A vegetação predominante é o cerrado, que apresenta fisionomias de campo limpo, campo sujo, cerrado, cerradão, mata ciliar, veredas e áreas de tensão ecológica, representadas pelo contato cerrado/floresta mesófita semidecídua e áreas de formação antrópicas que representam a ocupação de aproximadamente $70 \%$ do território. Nestas áreas há atividades agro-pastoris, com plantio de culturas cíclicas e pastagens. No perímetro urbano, durante o período de ocupação, houve a retirada da cobertura vegetal autóctone, sendo substituída, posteriormente, durante a arborização, por espécies nativas e exóticas. Em faixa ciliar, ao longo dos corpos d'água, localizada na área urbana, nota-se a presença de extensas áreas ocupadas por buritizais, formando veredas, e de alguns remanescentes florestais, destacando-se o Jardim Botânico, a Reserva Biológica da Universidade Federal de Mato Grosso do Sul (UFMS), a Reserva das Forças Armadas e a Reserva Ecológica do Parque dos Poderes, entre outros ${ }^{22}$.

Métodos de captura. Foram escolhidas doze estações para o monitoramento: Planalto e São Francisco na área central; Nova Campo Grande, São Conrado e Indubrasil no setor oeste; Rita Vieira no setor leste; Nova Lima, Monte Castelo e Veraneio no setor norte; Monte Alegre, Marcos Roberto e Centro Oeste no setor sul (Figura 1). Em cada estação, quatro residências foram selecionadas para colocação das armadilhas luminosas tipo CDC. Em cada residência eram colocadas duas armadilhas, uma no intradomicílio e outra no periodomicílio, a 1,5m de altura, uma vez ao mês. As armadilhas eram expostas das 16:00h às 7:00h da manhã seguinte; no período de maio de 2003 a abril de 2005 , totalizando 24 meses.

Devido à intensa transmissão da doença, algumas estações onde os casos humanos estavam mais concentrados (Planalto, Monte Castelo, São Francisco e Marcos Roberto), foram borrifadas com alphacypermetrina. Foram utilizadas máquinas costais e a borrifação foi realizada no interior e exterior das residências, incluindo anexos. Os resultados foram analisados pelo teste de McNemar.

Identificação das espécies. Os exemplares capturados foram mortos com clorofórmio, fixados em álcool $70 \%$ e, a seguir, preparados e montados em lâmina com Berlese. Os espécimes foram identificados de acordo com a classificação proposta por Galatit ${ }^{11}$.

\section{RESULTADOS}

No período entre maio de 2003 e abril de 2005 , foram capturados 2.275 flebotomíneos, destes 2.115 (92,9\%) da espécie Lutzomyia longipalpis e 160 de outras espécies (Tabela 1).

Somente a espécie Lutzomyia longipalpis, vetora da leishmaniose visceral, foi objeto do estudo, não tendo sido consideradas outras espécies. A porcentagem de machos capturados $(79,4 \%)$ foi maior que a das fêmeas $(20,6 \%)$.

A abundância relativa de Lutzomyia longipalpis foi maior no peridomicílio, com exceção dos meses de setembro e outubro de 2003 , julho de 2004 e janeiro de 2005 , sendo os valores mensais mostrados na Tabela 2. 0 índice médio da espécie Lutzomyia longipalpis por estações de captura e por armadilha no período é mostrado na Tabela 3. A densidade no período 


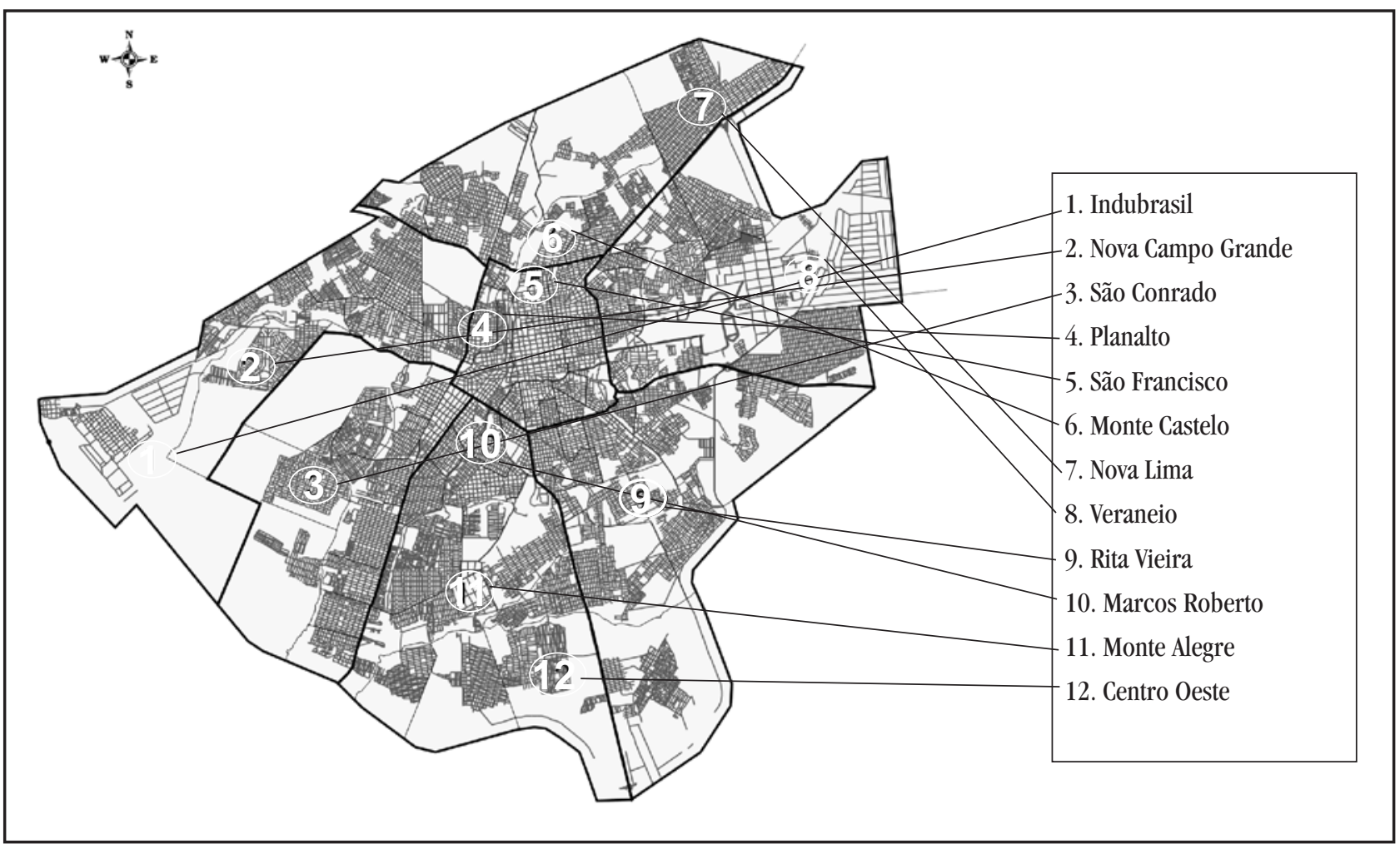

Figura 1 - Mapa do Município de Campo Grande, MS, com a localização das estações de captura dos flebotomíneos.

Tabela 1 - Espécies de flebotomíneos, conforme a classificação de Galati, 2003, coletados em armadilhas CDC, por sexo, em Campo Grande, MS, Brasil.

\begin{tabular}{lcc}
\hline Espécies & Masculino & Feminino \\
\hline Brumptomyia avellari & 1 & 0 \\
Brumptomyia spp & 0 & 1 \\
Evandromyia lenti & 10 & 6 \\
Evandromyia termithopila & 1 & 0 \\
Lutzomyia longipalpis & 1.679 & 436 \\
Lutzomyia renei & 1 & 0 \\
Micropygomyia peresi & 1 & 0 \\
Nyssomyia intermedia & 8 & 11 \\
Nyssomyia whitmani & 23 & 21 \\
Pintomyia christenseni & 0 & 1 \\
Pintomyia damascenoi & 1 & 0 \\
Psathyromyia aragaoi & 1 & 1 \\
Psathyromyia shannoni & 55 & 16 \\
\hline Total & 1.781 & 494 \\
\hline
\end{tabular}

foi de 1 flebotomíneo por armadilha, semelhante à encontrada por Oliveira cols ${ }^{20}$ (1,6 flebotomíneos por armadilha) e inferior à observada em outros locais do Estado como na Serra da Bodoquena (62,8 flebotomíneos por armadilha ${ }^{12}$.

As condições climáticas em Campo Grande, no período estudado, são mostradas na Figura 2. Nos meses chuvosos o número de espécimes aumentou e nos meses frios e secos, diminuiu. 0 número de espécimes de Lutzomyia longipalpis nas diferentes estações borrifadas diminuiu do primeiro ano para o segundo (Tabela 4) e, nas não borrifadas aumentou (Tabela 5), sendo significativa a diferença $(0,01<\mathrm{p}<0,02)$ pelo teste de McNemar.
Tabela 2 - Abundância relativa da espécie Lutzomyia longipalpis coletada nos babitats intra e peridomiciliar durante o periodo de maio de 2003 a abril de 2005 em Campo Grande, MS.

\begin{tabular}{lcc}
\hline Meses & Índice no intradomicílio & Índice no peridomicílio \\
\hline Maio & 0,19 & 5,36 \\
Junho & 0,88 & 0,94 \\
Julho & 0,00 & 0,14 \\
Agosto & 0,03 & 0,18 \\
Setembro & 0,83 & 0,38 \\
Outubro & 1,05 & 0,30 \\
Novembro & 0,39 & 1,06 \\
Dezembro & 0,27 & 0,57 \\
Janeiro & 0,62 & 1,47 \\
Fevereiro & 0,32 & 2,75 \\
Março & 0,57 & 2,45 \\
Abril & 0,51 & 1,26 \\
Maio & 0,21 & 0,53 \\
Junho & 0,02 & 2,40 \\
Julho & 0,36 & 0,09 \\
Agosto & 0,08 & 0,13 \\
Setembro & 0,13 & 0,42 \\
Outubro & 0,33 & 2,42 \\
Novembro & 0,25 & 1,48 \\
Dezembro & 0,25 & 3,85 \\
Janeiro & 0,92 & 0,60 \\
Fevereiro & 0,67 & 4,79 \\
Março & 0,63 & 0,92 \\
Abril & 0,48 & 3,81 \\
\hline
\end{tabular}

Abundância relativa: $\mathrm{n}^{\circ}$ de Lutzomyia longipalpis coletados (intra ou peri)/total de domicílios pesquisados (intra ou peri) 


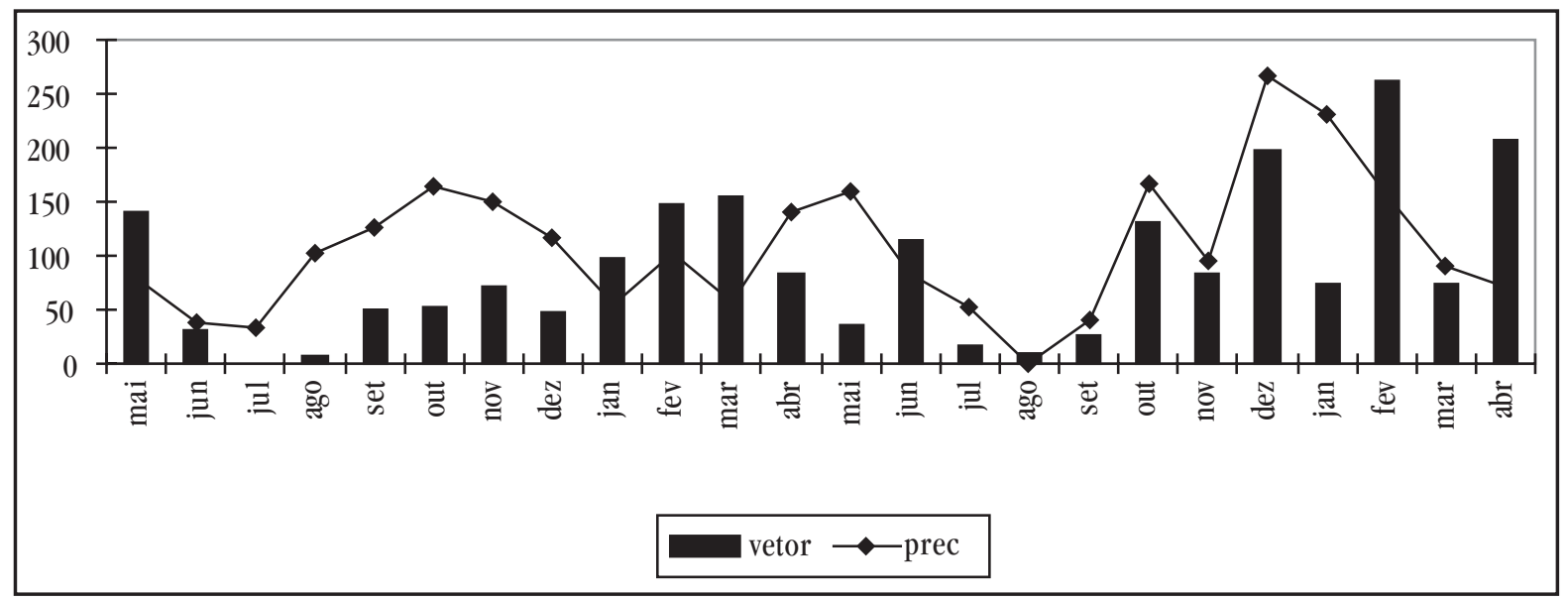

Figura 2 - Número de Lutzomyia longipalpis e precipitação pluviométrica, maio 2003 e abril de 2005, em Campo Grande, MS, Brasil.

Tabela 3 - Índice médio do flebotomíneo Lutzomyia longipalpis por estações, entre maio de 2003 e abril de 2005.

\begin{tabular}{lcc}
\hline Distritos & Estações & Índice por armadilha \\
\hline Oeste & Indubrasil & 0,27 \\
Oeste & Nova Campo Grande & 1,08 \\
Oeste & São Conrado & 1,88 \\
Central & Planalto & 1,20 \\
Central & São Francisco & 0,39 \\
Norte & Monte Castelo & 0,40 \\
Norte & Nova Lima & 0,02 \\
Norte & Veraneio & 0,20 \\
Leste & Rita Vieira & 2,32 \\
Sul & Marcos Roberto & 0,45 \\
Sul & Monte Alegre & 2,07 \\
Sul & Centro Oeste & 0,66 \\
\hline
\end{tabular}

Tabela 4 - Número de espécimes de Lutzomyia longipalpis capturados por período nas estações borrifadas, em Campo Grande, MS, Brasil

\begin{tabular}{lccc}
\hline Distritos & Estações & I período & II período \\
\hline Central & Planalto & 269 & 23 \\
Central & São Francisco & 21 & 46 \\
Norte & Monte Castelo & 53 & 24 \\
Sul & Marcos Roberto & 71 & 47 \\
\hline Total & & 414 & 140
\end{tabular}

0 I período compreende entre maio de 2003 e abril 2004 e o Il período entre maio de 2004 e abril 2005.

Tabela 5 - Número de espécimes de Lutzomyia longipalpis capturados por período nas estaç̃̃es não borrifadas, em Campo Grande MS, Brasil.

\begin{tabular}{lccc}
\hline Distritos & Estações & I período & II período \\
\hline Oeste & Indubrasil & 24 & 22 \\
Oeste & Nova C. Grande & 100 & 109 \\
Oeste & São Conrado & 69 & 271 \\
Norte & Nova Lima & 4 & 0 \\
Norte & Veraneio & 2 & 31 \\
Leste & Rita Vieira & 186 & 248 \\
Sul & Monte Alegre & 47 & 335 \\
Sul & Centro Oeste & 40 & 73 \\
\hline Total & & 472 & 1.089 \\
\hline
\end{tabular}

0 I período compreende entre maio de 2003 e abril 2004 e o II período entre maio de 2004 e abril 2005.

\section{DISCUSSÃO}

0 primeiro relato da presença de Lutzomyia longipalpis em Campo Grande, MS, foi feito em 2000 ${ }^{21}$, quando foram capturados 1.245 flebotomíneos e, destes, 71 eram Lutzomyia longipalpis. A espécie foi encontrada em dois ecótopos, um na região central da cidade e outro na região do Anhanduizinho, ambos na área urbana. Na época, não havia registro de casos humanos autóctones de LV, somente caninos. Foi observada uma mudança na fauna dos flebotomíneos entre o período de fevereiro de 1999 e fevereiro de 2000 e no período de maio de 2003 a abril de 2005, apesar das diferenças, em alguns aspectos, dos métodos de captura. Os locais de instalação das armadilhas no período entre 1999 e 2000 foram: na copa $(5 \mathrm{~m})$, solo e margem da mata $(1 \mathrm{~m})^{20}$ e no período entre 2003 e 2005, os locais escolhidos foram: nas residências $\left(1^{1 / 2} \mathrm{~m}\right)$ no intradomicílio e peridomicílio. A predominância da espécie Lutzomyia longipalpis em relação às outras espécies, após um período de quatro anos, mostra 0 alto grau de adaptação a ambientes urbanos. Esta predominância também foi observada em Porteirinha, $\mathrm{MG}^{3}$, em Jequié, $\mathrm{BA}^{8}$, e em Belo Horizonte, $\mathrm{MG}^{24}$.

De acordo com o Ministério da Saúde ${ }^{18}$, ao final da década de 80 , verificou-se a adaptação deste vetor aos ambientes urbanos, em periferias de grandes centros, principalmente na Região Sudeste, podendo ser encontrado no peridomicílio, e no intradomicílio.

No município de Campo Grande, MS, a porcentagem maior de machos capturados de Lutzomyia longipalpis com relação de 3,8:1 foi semelhante a valores encontrados por outros autores ${ }^{420}$. A abundância relativa foi maior no peridomicílio, principalmente em galinheiros, com exceção dos meses de setembro e outubro de 2003, julho de 2004 e janeiro de 2005. As estações com maior abundância foram Rita Vieira, Monte Alegre e São Conrado. São estações situadas próximas às nascentes e que possuem vegetação abundante. A estação Nova Lima, situada na porção nordeste da cidade, foi a que apresentou a menor quantidade do vetor, como mostrado na Tabela 3.

Inseticidas piretróides aplicados em paredes rebocadas e pintadas, padrão da grande maioria dos domicílios do município, 
possuem efeito residual de aproximadamente três meses e reduzem a população da espécie Lutzomyia longipalpis ${ }^{7}$.

Devido ao elevado número de casos humanos e caninos, algumas estações foram borrifadas com alphacypermetrina, com intervalos de quatro meses. Das quatro estações borrifadas, houve a diminuição da densidade do vetor nas estações Planalto, Monte Castelo e Marcos Roberto. Na estação São Francisco houve aumento (Tabela 4). Esta última é uma estação localizada na área central, com muitas construções verticais e onde houve índice elevado de recusa dos moradores em aceitar a prática de controle. Nas outras estações onde não ocorreu a borrifação observou-se aumento em cinco (Rita Vieira, São Conrado, Veraneio, Centro Oeste e Monte Alegre), e em três, manteve-se a mesma quantidade (Nova Lima, Nova Campo Grande e Indubrasil) (Tabela 5).

A borrifação é uma das medidas de controle que deve ser utilizada para o controle da leishmaniose visceral nas áreas com maiores índices do vetor e de maior ocorrência de casos humanos e caninos, os resultados desse período mostraram uma correlação positiva entre o uso da borrifação e a redução no número de vetores.

Em estudo realizado no Município de Corumbá, MS, observou-se que a população da espécie Lutzomyia longipalpis apresentou picos na primavera, verão e outono, meses chuvosos e de temperaturas elevadas ${ }^{13}$. Apesar de não ter havido correlação significativa entre precipitação pluviométrica e abundância de Lutzomyia longipalpis, observou-se aumento durante ou logo após períodos chuvosos (Figura 2).

A presença de Lutzomyia longipalpis em alta proporção no ambiente com relação às outras espécies de flebotomíneos, na presença de leishmaniose humana e canina, indica o papel desse inseto como o vetor responsável pela transmissão da LV em Campo Grande durante o período desses estudos.

\section{AGRADECIMENTOS}

Aos servidores da Fundação Nacional de Saúde, MS, da Secretaria de Saúde do Estado, MS, do Centro de Controle de Zoonoses, Prefeitura Municipal de Campo Grande que participaram deste trabalho e aos moradores de Campo Grande que permitiram a instalação das armadilhas em seus domicílios.

\section{REFERÊNCIAS}

1. Alves WA, Bevilacqua PD. Reflexões sobre a qualidade do diagnóstico da leishmaniose visceral canina em inquéritos epidemiológicos: o caso da epidemia de Belo Horizonte, Minas Gerais, Brasil, 1993-1997. Cadernos de Saúde Pública 20: 259-256, 2004 .

2. Andrade Filho JD, Silva ACL, Falcão AL. Phlebotomine sand flies in the state of Piauí, Brazil (Diptera: Psychodidae). Memórias do Instituto Oswaldo Cruz 96:1085-1087, 2001.

3. Barata RA, França-Silva JC, Meyrink W, Silva JC, Prata A, Lorosa ES, Fiúza JÁ, Gonçalves CM, Paula KM, Dias ES. Aspectos da ecologia e do comportamento de flebotomíneos em área endêmica de leishmaniose visceral, Minas Gerais. Revista da Sociedade Brasileira de Medicina Tropical 38: 421-425, 2005.

4. Barata RA, Silva JCF, Costa RT, Fortes-Dias CL, Silva JC, Paula EV, Prata A, Monteiro EM, Dias ES. Phlebotomine sand flies in Porteirinha, an área of american visceral leishmaniasis transmission in the State of Minas Gerais, Brazil. Memórias do Instituto Oswaldo Cruz 99: 481-487, 2004.

5. Bejarano EE, Uribe S, Rojas W, Velez ID. Phlebotomine sand flies (Díptera: Psychodidae) associated with the appearance of urban leishmaniasis in the city of Sincelejo, Colômbia. Memórias do Instituto Oswaldo Cruz 97: 645-647, 2002.

6. Dantas-Torres F, Brandão-Filho SP. Expansão geográfica da leishmaniose visceral no Estado de Pernambuco. Revista da Sociedade Brasileira de Medicina Tropical 39: 352-356, 2006.

7. De Silans, LNMP, Dedet JP, Arias, JR. Field monitoring of cypermethrin residual effect on the mortality rates of the phlebotomine sand fly Lutzomyia longipalpis in the State of Paraíba, Brazil. Memórias do Instituto Oswaldo Cruz 93: 39-344, 1998.

8. Dias-Lima AG, Guedes MLS, Sherlock IA. Horizontal stratification of the sandfly fauna (Díptera: Psychodidae) in a transitional vegetation between caatinga and tropical rain forest, state of Bahia, Brazil. Memórias do Instituto Oswaldo Cruz 98:733-737, 2003.

9. Empresa Brasileira de Pesquisa Agropecuária Gado de Corte. Relatório anual 2003-2005. Estação Meteorológica Embrapa - INMET. Campo Grande, MS, 2006.

10. Feitosa MM, Ikeda FA, Luvizotto MCR, Perri SHV. Aspectos clínicos de cães com leishmaniose visceral no município de Araçatuba-São Paulo (Brasil). Clínica Veterinária 28: 36-42, 2000.

11. Galati EAB. Classificação de Phlebotominae. In: Rangel EF, Lainson R (orgs) Flebotomíneos do Brasil. Fundação Oswaldo Cruz, Rio de Janeiro, p.53-126, 2003.

12. Galati EAB, Nunes VLB, Boggiani PC, Dorval MEC, Cristaldo G, Rocha HC, Oshiro ET, Gonçalves-Andrade RM, Naufel G. Phlebotomines (Díptera, Psychodidae) in caves of the Serra da Bodoquena, Mato Grosso do Sul, State, Brazil. Revista Brasileira de Entomologia 47: 283-296, 2003.

13. Galati EAB, Nunes VLB, Rego Jr FA, Oshiro ET, Chang MR. Estudo de flebotomíneos (Díptera: Psychodidae) em foco de leishmaniose visceral no Estado de Mato Grosso do Sul, Brasil. Revista de Saúde Pública 31: 378-390, 1997.

14. Instituto Brasileiro de Geografia e Estatística. www.ibge.gov.br/ cidadesat/default, Brasília, 2005.

15. Lainson R, Rangel EF. Lutzomyia longipalpis and the eco-epidemiology of American visceral leishmaniasis, with particular reference to Brazil: a review. Memórias do Instituto 0swaldo Cruz 100: 811-827

16. Margonari C, Freitas CR, Ribeiro RC, Moura ACM, Timbó M, Gripp AH, Pessanha $\mathrm{JE}$, Dias ES. Epidemiology of visceral leishmaniasis through spatial analysis, in Belo Horizonte municipality, state of Minas Gerais, Brazil. Memórias do Instituto Oswaldo Cruz 101: 31-38, 2006.

17. Marzochi MCA, Marzochi KBE. Tegumentary and visceral leishmaniases in Brazil - Emerging Anthropozoonosis and Possibilities for Their Control. Cadernos de Saúde Pública 10: 359-375, 1994.

18. Ministério da Saúde. Manual de vigilância e controle da leishmaniose visceral. Departamento de Vigilância Epidemiológica. Secretaria de Vigilância em Saúde, Ministério da Saúde. Brasília, 2003.

19. Missawa NA, Lima GBML. Distribuição espacial de Lutzomyia longipalpis (Lutz \& Neiva, 1912) and Lutzomyia cruzi (Mangabeira, 1938) no estado de Mato Grosso. Revista da Sociedade Brasileira de Medicina Tropical 39: 337-340, 2006

20. Oliveira AG, Andrade-Filho JD, Falcão AL, Brazil RP. Estudo de flebotomíneos (Díptera, Psychodidae, Phlebotominae) na zona urbana da cidade de Campo Grande, Mato Grosso do Sul, Brasil, 1999-2000. Cadernos de Saúde Pública 19: 933-944, 2003.

21. Oliveira AG, Falcão AL, Brazil RP. Primeiro encontro de Lutzomyia longipalpis (Lutz \& Neiva, 1912) na área urbana de Campo Grande, MS, Brasil. Revista de Saúde Pública 34: 654-655, 2000.

22. Prefeitura Municipal de Campo Grande. Perfil Sócio-Econômico de Campo Grande. Instituto Municipal de Planejamento Urbano, Campo Grande, MS, 1999.

23. Prefeitura Municipal de Campo Grande. Coordenadoria de Vigilância em Saúde. Serviço de Vigilância Epidemiológica. Sinan. Secretaria Municipal de Saúde, Campo Grande, MS, 2005. 
24. Resende MC, Camargo MCV, Vieira JRM, Nobi RCA, Porto NMN, Oliveira CDL, Pessanha JE, Cunha MCM, Brandão ST. Seasonal variation of Lutzomyia longipalpis in Belo Horizonte, State of Minas Gerais. Revista da Sociedade Brasileira de Medicina Tropical 39: 51-55, 2006.

25. Santos SO, Arias J, Hoffmann MP, Furlan MBG, Ferreira WF, Pereira C, Ferreira LS. The presence of Lutzomyia longipalpis in a focus of American visceral leishmaniasis where the only proven vector is Lutzomyia cruzi, Corumbá, Mato Grosso do Sul State. Revista da Sociedade Brasileira de Medicina Tropical 36: 633-634, 2003.

26. Santos SO, Arias J, Ribeiro AA, Hoffmann MP, Freitas RA, Malacco MAF. Incrimination of Lutzomyia cruzi as a vector of American Visceral Leishmaniasis. Medical and Veterinary Entomology 12: 315-317, 1998.
27. Silva ES, Oliveira AG, Carvalho FG, Silva EA, Friozi E, Farias, R. Primeiro relato de leishmaniose visceral canina em área urbana do município de Campo Grande, Mato Grosso do Sul. In: resumos do XXXVI Congresso Brasileiro de Medicina Tropical, São Luís, MA p. 318, 2000.

28. World Health Organization Seventeenth Programme Report/Progress 2003-2004. TDR 2005. www.who.int, 2005

29. Young D, Duncan M. Guide to identification and geographic distribution of Lutzomyia sandflies in Mexico, the West Indies, Central and South America (Diptera: Psychodidae). Memoirs of the American Entomological Institute 54: 1-881, 1994. 